\title{
Air-snow exchange of nitrate: a modelling approach to investigate physicochemical processes in surface snow at Dome $C$, Antarctica
}

\author{
Josué Bock et al. \\ Correspondence to: Josué Bock (josue.bock@meteo.fr)
}

The copyright of individual parts of the supplement might differ from the CC-BY 3.0 licence. 


\section{S1 Comparison between surface snow temperature deduced from longwave radiation measurement or modelled}

In-situ measurements of snow surface temperature and temperature profile are not straightforward. A convenient and usual way to get this variable is to use measured downwelling and upwelling longwave radiation and further applying Stefan-Boltzmann law to the snow considered as a "grey body":

$J_{\mathrm{LW} \uparrow}=\varepsilon \sigma T_{s}^{4}+(1-\varepsilon) E_{\mathrm{LW} \downarrow}$

where $J_{\mathrm{LW} \uparrow}$ and $E_{\mathrm{LW} \downarrow}$ are the upwelling and downwelling longwave radiation respectively, $\varepsilon$ is the snow emissivity (0.97 or 0.98 at DC, Town et al. (2008) and ref. therein) $\sigma$ is the Stefan Boltzman constant, and $T_{s}$ is the surface temperature.

Downwelling data is provided by the baseline surface radiation network (BSRN, see for example Vitale, Vito, 2009). Upwelling data were provided by Christian Lanconelli (personal communication). We present here the time series for only 2 weeks, but the following analysis was applied to the whole 3 month period for which we got the longwave, upwelling raw data, excluding $\sim 8$ days of wrong or missing data.

As once can see in Fig. S1 the modelled temperature shows a warm bias, with an initial RMSE between modelled and calculated temperature equals to $3.5 \mathrm{~K}$. Applying a constant reduction of $-2.65 \mathrm{~K}$ to the modelled temperatures (dashed green line) leads to a better fit, with a RMSE reduced to $2.4 \mathrm{~K}$. However, it further appears that diurnal amplitude of the modelled temperature is somewhat smaller than the calculated temperature amplitude. It is possible to correct this by applying a linear relation to get a modified temperature $T^{\prime}$ :

$T^{\prime}=a \times T+b$

where $T$ is the original modelled temperature, and $a$ and $b$ are adjusted to minimize RMSE. The optimum fit with calculated temperatures is obtained with $a=1.1825$ and $b=$ $-42.9 \mathrm{~K}$ (dashed purple line in Fig. S1). The RMSE is reduced to $2.0 \mathrm{~K}$.

However, as discussed in the main text (see Sect. 2.2.1), since this comparison between modelled and calculated temperatures could not be made over the whole year, we decided to keep the modelled temperatures unchanged when using them as input in our model. Indeed, the longwave radiation data covers only the summer period, during which the sunlight is maximum. Thus, the discrepancy between modelled and measured temperatures might not be the same over the whole year. However, this comparison shows that great care should be devoted to snow surface temperature measurements.

\section{S2 Monthly discrepancy analysis}

In Table S1 is presented a monthly analysis of the model performance. The model is run in configuration 1 (solid state diffusion inside a layered grain) with boundary condition BC3 (physically-based, prognostic parameterisation of the co-condensation process). In this analysis, a linear regression was used, with the intercept forced at the origin.

\section{References}

Town, M. S., Waddington, E. D., Walden, V. P., and Warren, S. G.: Temperatures, heating rates and vapour pressures in near-surface snow at the South Pole, Journal of Glaciology, 54, 487-498, doi $10.3189 / 002214308785837075,2008$.

Vitale, Vito: Basic measurements of radiation at Concordia Station (2009-02), 2009. 


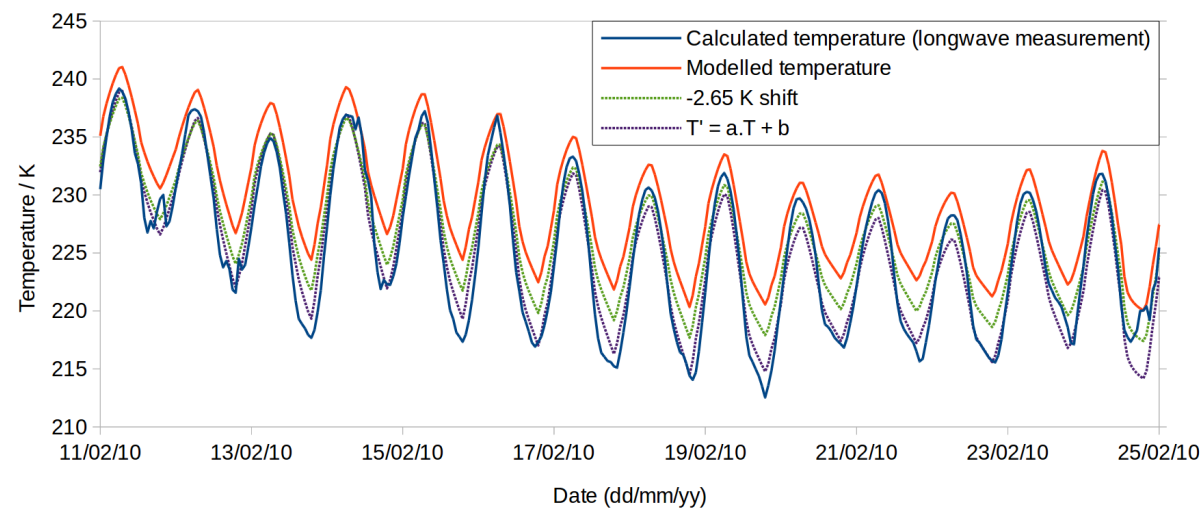

Figure S1. Surface snow temperature deduced from longwave radiation (solid blue line) and modelled temperature used in this study (solid red line). Modelled temperature offset by $-2.65 \mathrm{~K}$ is plotted in dashed green line. Modelled temperature modified using a linear function to increase its amplitude (see text) is plotted in dashed purple line.

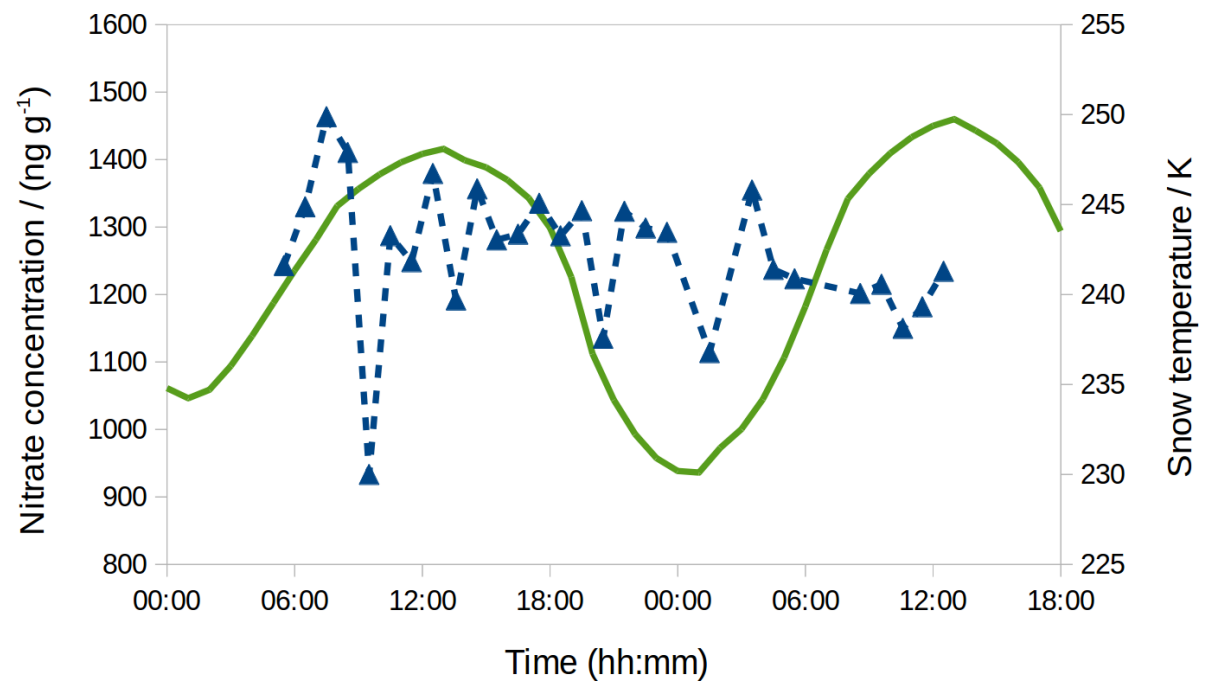

Figure S2. Hourly variations of skin layer nitrate concentration (blue triangles) at DC on 28 and 29 December 2009, and modelled snow surface temperature (solid green line).

Table S1. Here are summarised, for each month, the number of data points and the coefficient of determination $\left(\mathrm{R}^{2}\right)$ obtained using a linear regression with an intercept forced at the origin.

\begin{tabular}{clcc}
\hline Year & Month & \# points & $\mathrm{R}^{2}$ \\
\hline \multirow{4}{*}{2009} & February & 8 & 0.93 \\
& March & 9 & 0.95 \\
& April & 10 & 0.99 \\
& May & 9 & 0.91 \\
& June & 9 & 0.97 \\
& July & 14 & 0.92 \\
& August & 10 & 0.99 \\
& September & 12 & 0.84 \\
& October & 11 & 0.88 \\
& November & 12 & 0.87 \\
& December & 15 & 0.97 \\
2010 & January & 11 & 0.99 \\
\hline Whole dataset & 130 & 0.94 \\
\hline
\end{tabular}

